\title{
The Effect of Various Bur Types, Bond- ing Agents, and Composite Types on The Microleakage of Resin Composites
}

\author{
Department of Conservative Dentistry \\ College of Dentistry, University of Mosul
}

\begin{abstract}
الملاصة
الأهداف: تهدف هذه الدراسة إلى ثقييم تأثير أنواع محفرات السن والمواد اللاصقة وأنواع المركبات الراتنجية على التسرب المجهري لمركبات الراتنج في منطقتي الاتصال

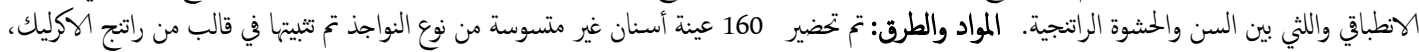

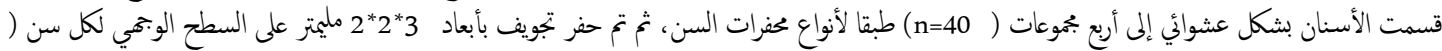

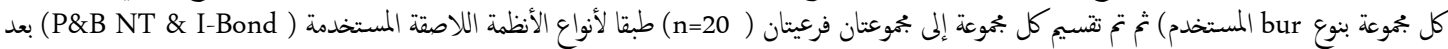

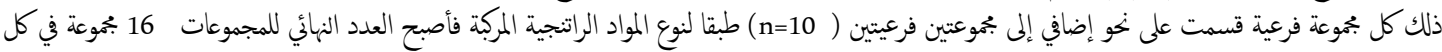

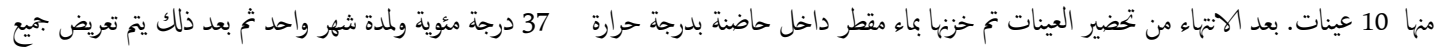

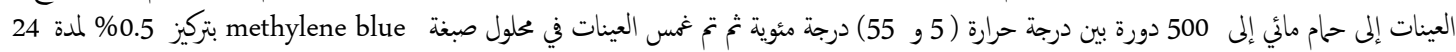

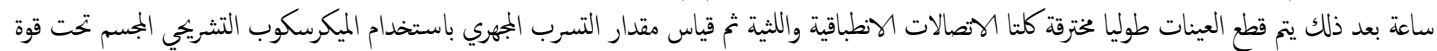

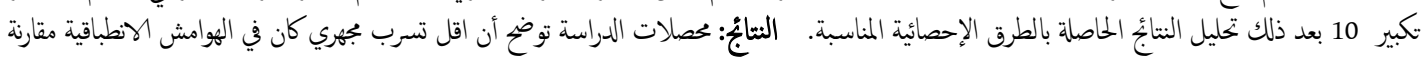

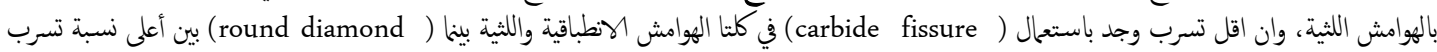

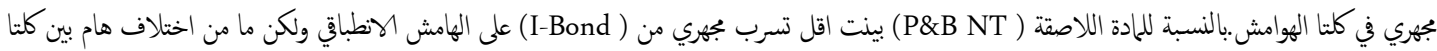

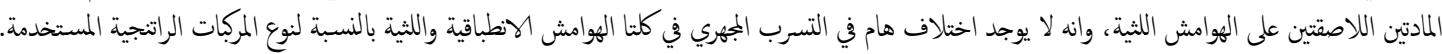

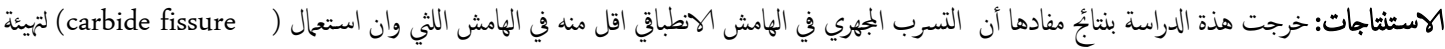

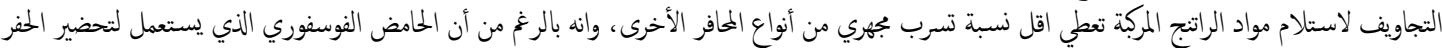

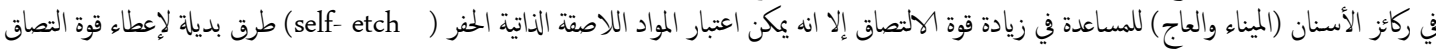

\begin{abstract}
Aims: This in vitro study was conducted to investigate the effect of various bur types, bonding agents, and resin composite restorative materials on the microleakage at occlusal and gingival tooth/composite interfaces. Materials and methods: One hundred and sixty non carious, extracted human premolars were mounted in acrylic resin, the teeth were randomly divided into four groups $(n=40)$ according to the bur types (diamond round bur, diamond fissure bur, carbide round bur, and carbide fissure bur). A standardize class $\mathrm{V}(3 \times 2 \times 2) \mathrm{mm}$ dimensions cavity was prepared on the facial surface of each tooth (each group with corresponding bur type), then each group was divided into two subgroups (n=20) according to the type of adhesive systems used (P\&B NT,\& I-Bond), after that each subgroup farther divided in to two subgroups $(n=10)$ according to the type of composite restorative materials (Arabesk $\& \mathrm{Tg}$ ) the final number of groups were (16) of (10) teeth in each. After the specimens were finished they stored for one month in $37^{\circ} \mathrm{c}$ distilled water, thermo cycled for 500 cycles between $\left(5^{\circ} \mathrm{c} \& 55^{\circ} \mathrm{c}\right)$ and immersed in $0.5 \%$ methylene blue solution for $24 \mathrm{~h}$., and then sectioned longitudinally. For both occlusal and gingival margins, dye penetration at the tooth/composite interfaces were scored from 0-3 under stereomicroscope at a magnification x10, data were analyzed using unpaired T-test, ANOVA, and Duncan's multiple rang test at 5\% significant level. Results: T-test represent that there was less microleakage at occlusal margins than the gingival margins. ANOVA and Duncan's multiple rang tests show that the less leakage was occurred with the use of carbide fissure bur at both occlusal and gingival margins while the diamond round bur show the highest leakage at both margins. P\&B NT show less leakage than I-Bond at the occlusal margin but there was no significant difference between P\&B NT \& I-Bond at the gingival margins also there was no significant difference between the (Arabesk \& Tg ) restorations at both occlusal and gingival margins. Conclusions: Within the limitations of this study results show that there was less microleakage at the occlusal margin than that at the gingival margins, and the use of carbide fissure bur to prepare the cavities for receiving resin composite materials show
\end{abstract}


the least microleakage than other type of burs, and although phosphoric acid has been intensely used to etch the dental substrates (enamel and dentin) for providing a good bonding, self-etching adhesives can be considered an alternative methods to provided a good bonding for restorative procedures.

Key Words: Dental burs, bonding agents, resin composite, microleakage.

Al-Askry RA. The Effect of Various Bur Types, Bonding Agents, and Composite Types on Microleakage of Resin Composite. Al-Rafidain Dent J. 2010; 10(1): 176-185 .

\section{INTRODUCTION}

It is important for the longevity of resin composite restorations that the formation of marginal gaps and cracks can be prevented or at least controlled to the greatest degree possible, if this could be achieved, it would be possible to minimize microleakage and its consequences, such as post operative sensitivity, pulp inflammation and caries recurrence, which are known to jeopardize the clinical longevity of the restoration ${ }^{(1,2)}$.

Cavity preparation with dental burs can easily produce micro- fracture in the enamel and the degree of the damage induced during cavity preparation can influenced by size and type of the burs. Cavity preparation with minimal mechanical damage at the cavosurface margins may be important factor for the preventing enamel cracks at the site of resin composite restoration $^{(3,4,5)}$.

The studies that investigated the surface characteristics of dentine found a significant difference in the roughness of surface prepared with different rotary instruments including diamond, tungsten carbide and tungsten carbide finishing burs ${ }^{(6,7)}$.

Dentinal surface morphology and smear layer formation are the main factors in the successful union of dental structure and restoration ${ }^{(3,8)}$.

There has always been a keen interest in the adaptation of dental restorative materials to the wall of the cavity and the retentive ability of a material to seal the cavity against ingress of oral fluids and microorganisms $^{(9)}$.

New adhesive systems continually have been improved to obtain an effective sealing at tooth/restoration interfaces, the recent trend in adhesive products is to simplify the process into two-steps or even one (single-step). Two-step total etch ad- hesive systems and self-etch adhesives were introduced in to the market in the mid 1990s, and late 2002 respectivly ${ }^{(10,11)}$.

In two-step total etch systems $\left(5^{\text {th }}\right.$ generation) a separated etch and rinse phase is still involved but a hydrophilic primer and hydrophobic resin are combined in to one application $^{(12)}$.

Self-etch adhesives or all-in-one $\left(7^{\text {th }}\right.$ generation) represent an alternative approach in enamel-dentin bonding, this system combines etching, priming, and adhesive potentials into a single step. They do not require a separated acid etch step which is not visibly controble by the dentist and are based on the use of non rinse acidic monomers that simultaneously condition and prime dentin and enamel ${ }^{(13,14)}$.

The use of self-etching adhesive systems has increased remarkably in clinical practice due to technique simplification and reduction of the number of clinical steps in addition, total-etch adhesive systems may produce imperfections in the hybrid layer due to dentin dehydration because acid etching and drying of the cavity are critical points of the bonding protocol $^{(15-17)}$.

Polymerization contraction stress is still one of the major problems when restoring teeth with resin composite since a contraction forces can be created and may disrupt the bond to cavity walls or create stresses to surrounding tooth structure and may result of tooth cracks or fractures. The Polymerization shrinkage and the viscosity were found to be a significant determinants of gap formation around resin composite $^{(1,18,19)}$.

The purpose of this in vitro study was to evaluate the effect of certain variables, including bur types, adhesive systems, and types of resin composite restorative mate- 
rials on the microleakage at the restoration/tooth structure interfaces.

\section{MATERIALS AND METHODS}

A total of one hundred sixty extracted human premolar teeth free of cracks (examined by fiber optic light), free of caries and restorations on the visual inspection were selected for experiment, all teeth were cleaned and polished using scalers and pumice, then stored in distilled water at room temperature prior to the use.

The root apex of each tooth was sealed with a sticky wax, two coats of nail varnish were applied to the entire external surface of the root, each tooth was mounted in a ring mold cold curing acrylic resin (Medicus, DMP Ltd.,Europe) to the level of cemento-enamel junction.

The specimens were randomly divided into four groups $(n=40)$ according to the type of dental bur used to prepared the cavity using high- speed handpiece with air/water coolant as follows:-

Group1: cavity preparation using a diamond round burs No.1014 (KG Sorensen Ind com. Ltd., Brazil).

Group2: cavity preparation using a diamond straight fissure burs No.1092 (KG Sorensen Ind com. Ltd., Brazil).

Group3: cavity preparation using a tungsten carbide round burs No.3 (Mani Inc. Japan).

Group4: cavity preparation using a tungsten carbide straight fissure burs No.56 (Mani Inc. Japan).

A standard class $\mathrm{V}$ cavities were prepared with the mentioned burs each according to its group with $3 \mathrm{~mm}$ long, 2 $\mathrm{mm}$ wide, and $2 \mathrm{~mm}$ in depth and placed on the facial surface of each tooth. Class V cavities prepared with the gingival margin $1.5 \mathrm{~mm}$ above the CEJ (completely bordered by enamel), cavosurface walls were finished to a butt joint to standardize the cavities (each 1 bur was used to prepare five cavities) $)^{(2)}$.

Following cavities preparation, specimens in each group were randomly divided into two group $(n=20)$ according to the type of adhesive system used as follows:

Group I: each prepared tooth was etch with $37 \%$ phosphoric acid gel (Vivdent Ets, Schaan/Liechtenstein, Germany) for
15 seconds, rinsed for 20 seconds, then gently blown to remove excess water (maintain a moist surface), a total etch nanofilled acetone-based adhesive system (Prime\&Bond NT, Dentsplay Detrey / Germany) was applied according to the manufacturers instructions.

Group II: a one-step self-etch adhesive system (I-Bond, Medicinos Linija UAB., EU) was applied on dried enamel and dentin surfaces according to the manufactures instructions.

Then the specimens in each sub-group were randomly divided into two sub-group $(n=10)$ according to the type of resin composite restorative materials used to fill the cavities as follows.

Group A: light-curing universal microhybrid composite with sintraglass multifiller system (Arabesk (A2), Voco, Germany).

Group B: fine glass hybride light cure composite (Tg (A2), Technical \& General Ltd, London, England).

The restorations were placed in two increments with each layer not being more than $1 \mathrm{~mm}$ thickness and cured for 40 second at $400 \mathrm{~mW} / \mathrm{cm}^{2}$ using a QuartzTungsten-Halogen (QTH) light curing unit (Astralis 5, Vivadent Schaan/Liechenstein, Germany), the radiometer was used to check the light efficiency before starting each restoration, then the restorations were finished and polished with Sof-Lex disks (3M,USA). Then all teeth were stored in distilled water at $37^{\circ} \mathrm{c}$ for one month. After this the teeth were thermocycled 500 time at a temperature of $5^{\circ} \mathrm{c} \pm 2^{\circ} \mathrm{c}$ and $55^{\circ} \mathrm{c} \pm 2^{\circ} \mathrm{c}$, with dwell times of 60 second in each water bath and 60 second transfer time between the baths ${ }^{(1)}$.

For all specimens the entire tooth surface were painted with two coats of nail varnish except for an area of $1 \mathrm{~mm}$ a way from the cavosurface margins to prevent dye penetration anywhere other than the restoration margins.

The samples were immersed in $0.5 \%$ methylene blue solution at $37^{\circ} \mathrm{c}$ for $24 \mathrm{hs}{ }^{(10)}$. Then the teeth were rinsed in running water, dried and then sectioned buccolingually at the center of the cavity using a diamond wheel saw (KG Sorensen SP, Brazil). Dye penetration at the occlusal and gingival margin was examined 
blind and independaly by two evaluators using a stereomicroscope (Hamilton by AITAY International Italy) at a magnification level of x10 scored according to the following criteria as described by Santini etal $^{(20)}$.

0: no dye penetration (Figure1).

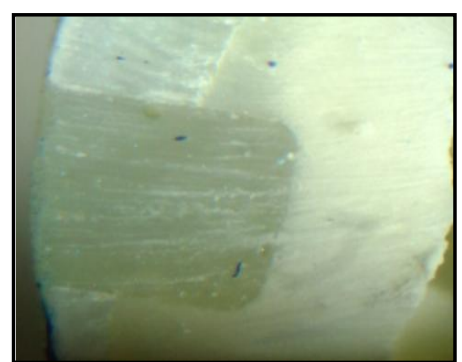

Figure(1): Sectioned tooth represent no dye penetration.

1: dye penetration to less than the half of occlusal or gingival wall (Figure.2).

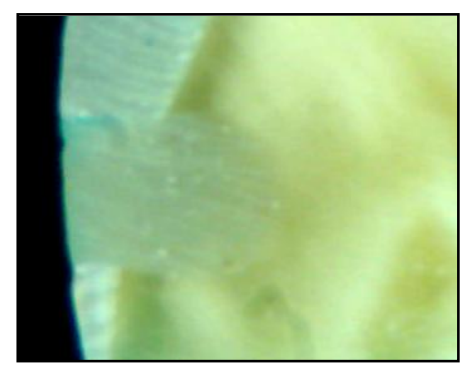

Figure.(2): Sectioned tooth represent score (1) of dye penetration

2: dye penetration along the occlusal or gingival wall but not including the axial wall(Figure.3).

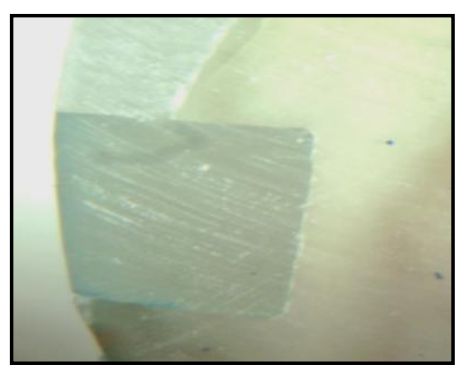

Figure(3): Sectioned tooth represent score (2) of dye penetration
3: dye penetration to and along the axiall wall (Figure.4).

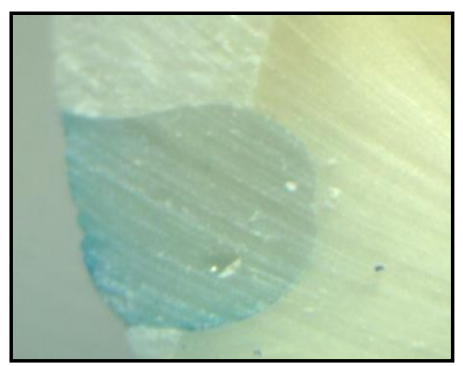

Figure.(4): Sectioned tooth represent score (3) of dye penetration.

The data was subjected to statistical Analysis to compare the microleakage at the occlusal and gingival walls using unpaired T-test at $(\mathrm{p}<0.05)$, analysis of variance (ANOVA) was conducted to find if the was a significant differences in the leakage among the groups at the occlusal and gingival margins at $(\mathrm{p}<0.05)$, then if there was a significant differences among the groups Duncan's multiple range test has been done at $(\mathrm{p}<0.05)$.

\section{RESULTS}

The leakage scores for different variables of all restorations at occlusal and gingival walls are described in table(1).

The mean values and standard deviations of microleakage scores of all groups at the occlusal and gingival walls are presented in table (2).

The mean values and standard deviations of microleakage scores of all groups at the occlusal and gingival walls are presented in table (2). Unpaired T-test disclosed a higher dye penetration at the gingival margins $(1.338, \pm 0.8233)$ than the occlusal margins $(1.075, \pm 0.7814)$ at $(\mathrm{p}<0.05)$. 
Table(1): The score of dye penetration for all groups at occlusal and gingival walls.

\begin{tabular}{ccccccccc}
\hline Groups & \multicolumn{3}{c}{ Occlusal } & \multicolumn{5}{c}{ Gingival } \\
& $\mathbf{0}$ & $\mathbf{1}$ & $\mathbf{2}$ & $\mathbf{3}$ & $\mathbf{0}$ & $\mathbf{1}$ & $\mathbf{2}$ & $\mathbf{3}$ \\
\hline G1,I,A & 1 & 5 & 4 & 0 & 0 & 4 & 5 & 1 \\
G1,I,B & 1 & 4 & 5 & 0 & 0 & 4 & 4 & 2 \\
G1,II,A & 0 & 4 & 6 & 0 & 0 & 3 & 6 & 1 \\
G1,II,B & 0 & 3 & 4 & 3 & 0 & 3 & 6 & 1 \\
G2,I,A & 3 & 4 & 3 & 0 & 1 & 2 & 5 & 2 \\
G2,I,B & 3 & 5 & 1 & 1 & 1 & 4 & 3 & 2 \\
G2,II,A & 0 & 6 & 4 & 0 & 0 & 3 & 6 & 1 \\
G2,II,B & 2 & 4 & 4 & 0 & 1 & 3 & 4 & 2 \\
G3,I,A & 3 & 7 & 0 & 0 & 2 & 6 & 2 & 0 \\
G3,I,B & 4 & 6 & 0 & 0 & 2 & 7 & 1 & 0 \\
G3,II,A & 2 & 5 & 3 & 0 & 1 & 5 & 3 & 1 \\
G3,II,B & 3 & 4 & 2 & 1 & 2 & 5 & 3 & 0 \\
G4,I,A & 5 & 5 & 0 & 0 & 3 & 7 & 0 & 0 \\
G4,I,B & 6 & 4 & 0 & 0 & 4 & 6 & 0 & 0 \\
G4,II,A & 3 & 5 & 2 & 0 & 3 & 5 & 2 & 0 \\
G4,II,B & 2 & 6 & 2 & 0 & 3 & 6 & 1 & 0 \\
\hline
\end{tabular}

G1: Diamond round bur; G2: Diamond fissure bur; G3:Carbide round bur; G4: Carbide fissure bur; I:P\&B NT; II: I-Bond; A: Arabesk; B:Tg

Table(2): The difference in mean and standared deviation values of microleakage between occlusal and gingival margins.

\begin{tabular}{lccc}
\hline Side of leakage & $\mathbf{N}$ & Mean & $\mathbf{\pm S D}$ \\
Occlusal Walls & 160 & 1.075 & 0.7814 \\
Gingival Walls & 160 & 1.338 & 0.8233 \\
\hline
\end{tabular}

$\mathrm{SD}=$ Standard deviation

Because there was a significant difference in microleakage between the occlusal and the gingival walls Analysis of Variance (ANOVA) for levels of bur types, bonding agents, and resin composite restorative materials and their interactions for the calculated data at the occlusal and the gingival margins had be done as shown in table (3) and (4).

Table(3):Analysis of variance for the bur types, bonding agents, resin composites, and their interactions at the occlusal margins

\begin{tabular}{|c|c|c|c|c|c|c|}
\hline Source of Variance & DF & $\begin{array}{l}\text { Sum of } \\
\text { Square }\end{array}$ & $\begin{array}{l}\text { Mean } \\
\text { Square }\end{array}$ & Cal.F & Tab.F & Sig. \\
\hline Bur types & 3 & 17.45 & 5.8167 & 11.797 & 2.60 & $0.05^{* *}$ \\
\hline Bonding Agent & 1 & 7.225 & 7.225 & 14.654 & 3,84 & $0.05^{* *}$ \\
\hline Resin composite types & 1 & 0.025 & 0.025 & 0.0507 & 3.84 & N.S \\
\hline Bur types $\times$ Bonding Agent & 3 & 0.225 & 0.075 & 0.1521 & 2.60 & N.S \\
\hline $\begin{array}{c}\text { Bur types } \times \text { Resin composite } \\
\text { types }\end{array}$ & 3 & 0.725 & 0.2417 & 0.4901 & 2.60 & N.S \\
\hline $\begin{array}{l}\text { Bonding Agent } \times \text { Resin com- } \\
\text { posite types }\end{array}$ & 1 & 0.1 & 0.1 & 0.2028 & 3.48 & N.S \\
\hline $\begin{array}{l}\text { Bur types } \times \text { Bonding Agent } \times \\
\text { Resin composite types }\end{array}$ & 3 & 0.35 & 0.1167 & 0.2366 & 2.60 & N.S \\
\hline Error & 144 & 71 & 0.4930 & & & \\
\hline Corrected Total & 159 & 97.1 & & & & \\
\hline
\end{tabular}

DF = Degree of Freedom; Cal.F: Calculated F.value; Tab.F: Tabulated F.value; Sig: Significance; Highly Significant; N.S: Not Significant 
Table(4):Analysis of variance for the bur types, bonding agents, resin composites, and their interactions at the gingival margins.

\begin{tabular}{lcccccc}
\hline \multicolumn{1}{c}{ Source of Variance } & DF & $\begin{array}{c}\text { Sum of } \\
\text { Square }\end{array}$ & $\begin{array}{c}\text { Mean } \\
\text { Square }\end{array}$ & Cal.F & Tab.F & Sig. \\
\hline Bur types & 3 & 29.725 & 9.908 & 18.823 & 2.60 & $0.05^{* *}$ \\
Bonding Agent & 1 & 0.9 & 0.9 & 1.7098 & 3,84 & N.S \\
Resin composite types & 1 & 0.4 & 0.4 & 0.7599 & 3.84 & N.S \\
Bur types $\times$ Bonding Agent & 3 & 0.45 & 0.15 & 0.285 & 2.60 & N.S \\
Bur types $\times$ Resin composite types & 3 & 0.35 & 0.1167 & 0.2216 & 2.60 & N.S \\
$\begin{array}{l}\text { Bonding Agent } \times \text { Resin composite } \\
\text { types }\end{array}$ & 1 & 0.025 & 0.025 & 0.0475 & 3.48 & N.S \\
$\begin{array}{l}\text { Bur types } \times \text { Bonding Agent } \times \text { Resin } \\
\text { composite types }\end{array}$ & 3 & 0.125 & 0.0417 & 0.0792 & 2.60 & N.S \\
Error & 14 & 75.8 & 0.5263 & & & \\
Corrected Total & 4 & & & & & \\
\hline
\end{tabular}

DF: Degree of Freedom; Cal.F: Calculated F.value; Tab.F: Tabulated F.value; Sig: Significance; Highly Significant; N.S: Not Significant

ANOVA for microleakage data at the occlusal walls showed that there was a significant differences in microleakage at bur types and bonding agents levels (p>0.05). Duncan's multiple rang test was carried out to determined where those dif- ferences occurred. For comparing four types of burs the least leakage was detected for fissure carbide bur groups $(0.7$, \pm 0.648 ), while round diamond bur groups showed the highest leakage $(1.575, \pm 0.72)$ at $(\mathrm{p}<0.05)$ table $(5)$.

Table(5): Duncan's Multiple Range Test for the Effect of bur types on the microleakage at occlusal margin.

\begin{tabular}{ccccc}
\hline Bur Types & N & Mean & $\mathbf{\pm S D}$ & Duncan's Grouping \\
\hline Round diamond bur & 40 & 1.575 & 0.721 & D \\
Fissure diamond bur & 40 & 1.15 & 0.769 & C \\
Round carbide bur & 40 & 0.875 & 0.722 & B \\
Fissure carbide bur & 40 & 0.7 & 0.648 & A \\
\hline
\end{tabular}

Mean with different letters are statistically different.

Duncan's multiple rang test was carried out to comparing the two types of bonding agents at $(\mathrm{p}<0.05)$, Prime\&Bond NT groups showed less leakage $(0.862$, $\pm 0.724)$ than I-Bond groups (1.287, $\pm 0.782)$ as shown in table(6).

Table(6): Duncan's Multiple Range Test for the Effect of bonding agent types on the microleakage at occlusal margin.

\begin{tabular}{ccccc}
\hline Adhesive types & N & Mean & \pm SD & $\begin{array}{c}\text { Duncan's Group- } \\
\text { ing }\end{array}$ \\
\hline P\&B NT & 80 & 0.862 & 0.724 & $\mathrm{~A}$ \\
I-Bond & 80 & 1.287 & 0.782 & $\mathrm{~B}$ \\
\hline
\end{tabular}

Mean with different letters are statistically different.

ANOVA for microleakage data at gingival margins (table 4) shows that there was a significant differences in microleakage at bur types level only $(\mathrm{p}>0.05)$. Duncan's multiple rang test revealed that the least leakage was detected for fissure carbide bur groups $(0.75, \pm 0.588)$, while round diamond bur groups showed the highest leakage $(1.775, \pm 0.659)$ table $(7)$. 
Table(7): Duncan's Multiple Range Test for the Effect of bur types on the microleakage at gingival margin.

\begin{tabular}{ccccc}
\hline Bur Types & N & Mean & $\mathbf{\pm S D}$ & Duncan's Grouping \\
\hline Round diamond bur & 40 & 1.775 & 0.659 & $\mathrm{C}$ \\
Fissure diamond bur & 40 & 1.725 & 0.846 & $\mathrm{C}$ \\
Round carbide bur & 40 & 1.1 & 0.708 & $\mathrm{~B}$ \\
Fissure carbide bur & 40 & 0.75 & 0.588 & $\mathrm{~A}$ \\
\hline
\end{tabular}

Means with different letters are statistically different.; Means with similar letters are statistically not different.

The result in this study showed no significant differences in microleakage between the type of composite restorations ( Arabesk \& $\mathrm{Tg}$ ) at both occlusal and gingival marginal ridges.

\section{DISCUSSION}

Three variables related to microleakage were investigated in this study, the effect of various bur types, the type of bonding agent used, and the type of resin composite restorative materials.

In the present study, higher leakage was detected at the gingival margins when comparing to occlusal margins.

The results show that carbide bur revealed less microleakage than diamond bur at the occlusal and the gingival walls. Studies showed that carbide burs leave cleaner surface than diamond burs, and it gave thinnest smear layer when comparing with other types of preparations ${ }^{(3,8,21)}$.

Preparing a dentinal surface with diamond bur decrease the dentinal permeability compared to a carbide bur, presumably because of the collection of smear layer in addition to the different thickness of smear layer that created by diamond burs. It is expected that smear layer morphology and quality will vary considerably according to the different methods used in preparing the surface ${ }^{(4,7)}$.

Studies showed that during preparation with diamond burs the enamel get damage and cracks are formed and distributed a long the boundaries between the enamel rod. It is suggested that the action of the mechanism of this instruments is responsible for the microleakage since chipping out pieces of enamel has been occurred $(1,3)$
Studies revealed that fissure burs revealed less leakage than round bur and this may be due to the action of the bur (Figure.5a-d), on the other hand the heat generation and the type of grooves formed during preparation could lead to inclusion of air bubbles between the enamel and the adhesive $e^{(1,4,6)}$.

A critical analysis of cutting instruments used in the preparation of cavity revealed that when it comes to removing enamel and dentine, diamond instruments proved to be the most effective of all. However, it is presumed that surface registry and irregularity promote the wetability by producing increased surface area and that the bond between the adherent surface and the adhesive will be subsequently stronger. However, other studies showed that excessive roughness may hinder the even flow of the liquid adhesive and numerous voids were exposed at the interface in addition to the structural faults as represent in (Fig.6a,c) $)^{(6,11,21)}$.

Analysis of variance for data collected at the occlusal walls shows that P\&B NT adhesive revealed less leakage than I-bond adhesive.

Many studies show that self-etching adhesives could seal enamel margins effectively. However, in other studies selfetching adhesives showed greater scales of microleakage than adhesives using conventional phosphoric acid as etchant $^{(2,10,11,18,22)}$.

Studies have shown that in enamel etched by $37 \%$ phosphoric acid the adhesive system penetrate to a depth of $10 \mu \mathrm{m}$ whereas in enamel etched by components of the self-etching adhesives the adhesive only reaches a depth of $0.6-0.7 \mu \mathrm{m}^{(11,16,17)}$. 

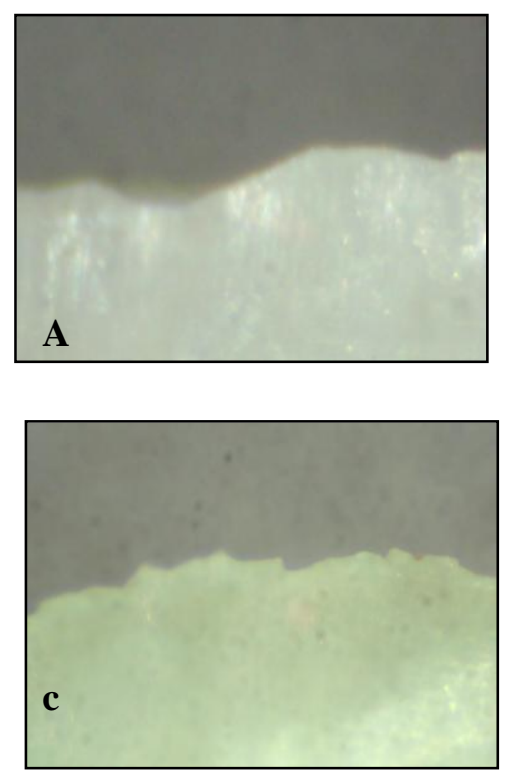

Figure (5): Stereomicroscopic images of the marginal enamel (x40).

A: Prepared with diamond round bur (group 1). B: prepared with diamond fissure bur (group 2); C: prepared with carbide round bur (group 3); d: prepared with carbide fissure bur (group 4).
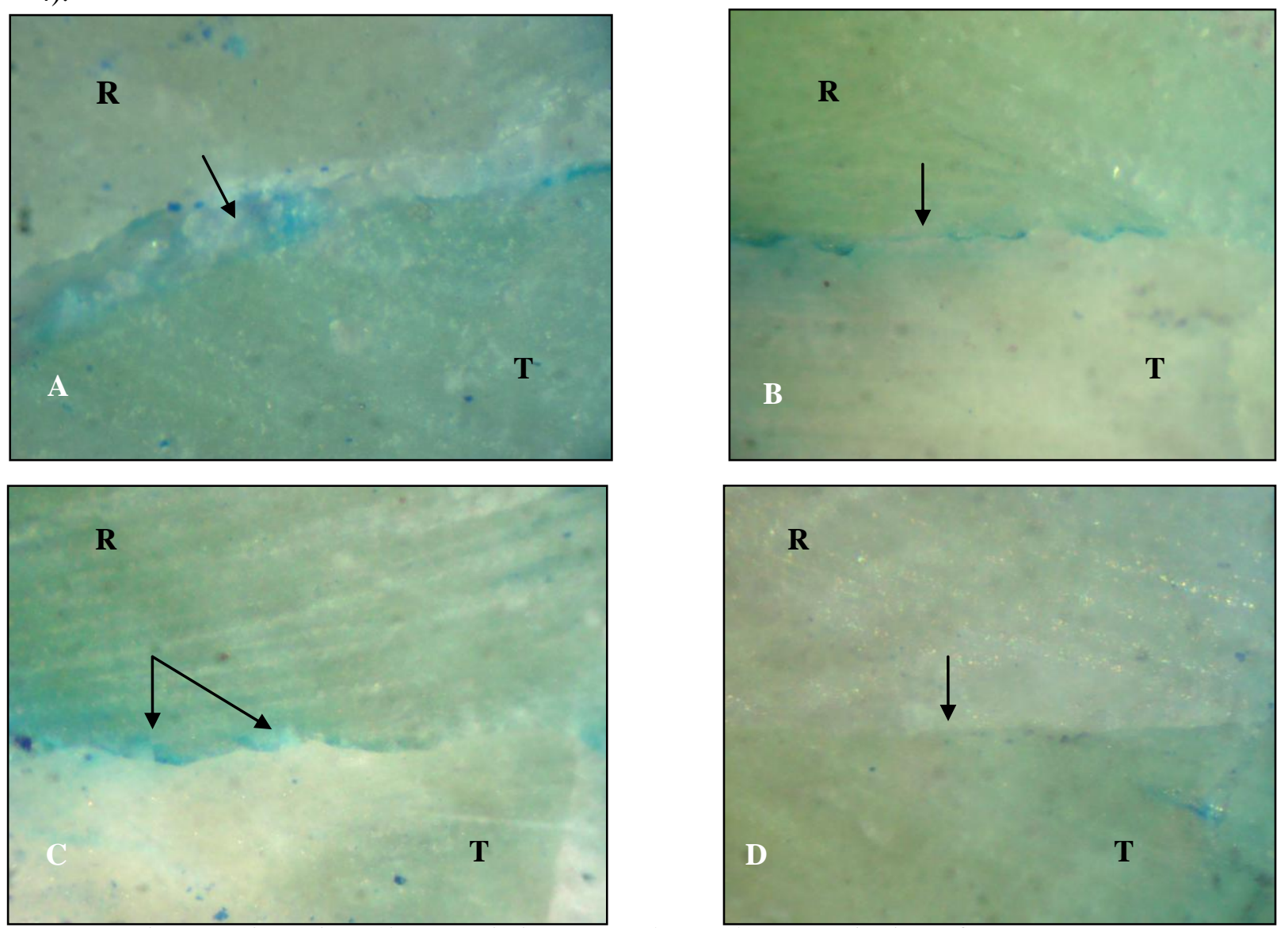

Fig.(6): Dissecting microscopic images at the tooth-composite inter face (x40).

T: Tooth structure; R: Resin composite; A: Prepared with diamond round bur (group 1). Arrow showed gap formation; B: Prepared with diamond fissure bur (group 2). Arrow showed the tooth-composite interface.; $\mathrm{C}$ : prepared with carbide round bur (group 3). Arrow showed small air void; D: prepared with carbide fissure bur (group 4). Arrow showed the toothcomposite interface. 
Etch and rinse adhesive systems remove the smear layer completely and expose the collagen fibrils while for selfetching primers the smear layer is not completely removed but incorporated into the hybrid layer. Since a primer and an adhesive can penetrate the surface better when the smear layer is removed it might play an important role in decreasing microleakage of composite resin restoration in these cavities ${ }^{(2,8-10,15,23)}$.

I-bond show higher scores of microleakage than $\mathrm{P} \& \mathrm{~B} \mathrm{NT}$, also this is may be due to lack of filler in its composition which lead to greater polymerization shrinkage of adhesive ${ }^{(22,23)}$.

On other side Analysis of Variance for data collected at the gingival margins represented that there was no significant difference between P\&B NT and I-Bond and this may be due to the fact that totaletch system completely removed the smear layer and open the dentinal tubules and dematerialized the subjacent dentin leaving exposed collagen network to be encapsulated after primer and bonding application forming a hybrid layer, but in some investigations represented that the bonding agent may does not completely penetrate the dentine leaving unprotect exposed collagen which can be degrade over time causing adhesive failure and microleakage ${ }^{(16,18)}$.

On other hand since P\&B NT adhesive containing nanofiller this filler some times makes it difficult to penetrate into interprismatic areas as deeply as unfilled adhesives so it has greater difficulty to penetrate enamel as much as the conditioning depth $^{(8,22)}$.

Enamel cracks is initiated by the damage caused during preparation with burs and is likely to be furthered by the contraction of the polymerizing resin composite $^{(1,19)}$. In this study statistical analysis showed no significant differences in microleakage between the two types of resin composites ( Arabesk \& Tg) and this may be due to the insertion technique used in this study also the use of same color shade which may be reduce the effect of colorants on light polymerization in addition to the use of same curing unit.

\section{CONCLUSIONS}

Within the limitation of this invitro study, it could be concluded that:

1- There was less leakage at occlusal walls than at gingival walls.

2- Selecting a bur for cavity preparations an important factor for improve the cavosurface margin integrity.

3- Although phosphoric acid has been intensely used to etch the dental substrates (enamel and dentin) for bonding, selfetching adhesives are consider alternative methods to prepare the tooth for restorative procedures.

\section{REFERENCES}

1. Nishimura K., Ikeda M., Yoshikawa T., Otsuki M., and Tagami J. Effect of various grit burs on marginal integrity of resin composite restorations. J Med Dent Sci 2005; 52:9-15.

2. De Araûjo CS., Da Silva TI., Ogliari FA., Meireles SS.,Piva E., and Demarco FF. Microleakage of seven adhesive system in enamel and dentin. J contem Dent Pract 2006;7:26-33.

3. Larson TD. A traumatic tooth preparation. J Minneso Dent Associ 2008;87:55-65.

4. Sevgican F., Inoue S., Koase K., Kawamoto C., Ikeda T., and Sano H. Bond strength of simplified-step adhesives to enamel prepared with two different diamond burs. Austra Dent J 2004;49:141-145.

5. Vieira ÁS., Dos Santos MP., Antunes LA., PrimoLG., and Maia LC. Preparation time and sealing effect of cavities prepared by an ultrasonic device and a high-speed diamond rotary cutting system. J Oral Sci 2007;49:207-211.

6. AL-Omari WM, Mitchell CA, and Cunningham JL. Surface roughness and wettability of enamel and dentin surface prepared with different dental burs. J Oral Rehabilit 2001;28:645-650.

7. Von Fraunhover JA., Smith TA., and Marshall KR. The effect of multiple uses of disposable diamond burs on restoration leakage. JADA 2005;136:53-57.

8. Oliveira SS., Pugach MK., Hilton JF., Watanabe LG., Marshall SJ., and Marshall GW. The influence of the dentin smear layer on adhesion: a self-etching primer vs. a total-etch system. Dent Mater 2003;19:758-767. 
9. Mali P., Shobha D., and singh A. Microleakage of restorative materials: An in vitro study. J Indian Soc Pedod Prev Dent 2006; 24:15-18.

10. Deliperi S. ,Bardwell DN., and Wegley C. Restoration interface microleakage using two total-etch and two self-etch adhesives. Oper Dent 2007;32:174-179.

11. Khosravi K., and Mousavi M. Effect of pre-etching on sealing ability of two current self-etching adhesives. J Res Med Sci 2005;10:150-155.

12. Francescantonio MD., Oliveira MT., Shinohara MS., Ambrosano GM., and Giannini M. Bond strength evaluation of self-etch and total-etch adhesive systems on intact and ground human enamel. Braz J Oral Sci 2007;6:1462-1466.

13. Dunn JR. I-Bond ${ }^{\mathrm{TM}}$ the seventh-generation, one-bottle dental bonding agent. Compend Contin Educ Dent 2003;24:14-18.

14. Owens BM., and Johnson WW. Effect of single step adhesives on the marginal permeability of class $\mathrm{V}$ resin composites. J Oper Dent 2007;32:67-72.

15. Susin AH., Alves LS., Melo GP., and Lenzi TL. Comparative scanning electron microscopic study of the effect of different dental conditioners on dentin micro morphology. J Appl Oral Sci 2008;16:100105.

16. Shinohara MS., Olivera MT., HipólitoVD., Giannini M., and Goes MF. SEM analysis of the acid-etched dental pattern promoted by acidic monomers and phosphoric acids. J Appl Oral Sci 2006;14:427-
435.

17. Moura SK., Santos JF., and Ballester RY. Morphological characterization of the tooth/adhesive interface. Braz Dent J 2006; 17:179-185.

18. De Munck J, Van Landuyt K, Peumans M, Poitevin A, Lambrechts P, Braem M, and Van Meerbeek B. A critical review of the durability of adhesion to tooth tissue: Method and Results. J Dent Res 2005;84:118132.

19. Ferracane JL. Developing a more complte understanding of stresses produced in dental composites during polymerization. Dent Mater 2005;21:36-42.

20. Santini A, Ivanovic V, Ibbetson R, and Milia E. Influence of marginal bevels on microleakage around class $\mathrm{V}$ cavities bonded with seven self-etcing agents. Am J Dent 2004; 17:257-264.

21. Yoshida E., Uno S., Nodasaka Y., Kaga M., Yawaka Y., and Hirano S. Effect of grinding surface on dentin bond strength of all-in-one adhesives. J Dent Mate 2006;25:47-53.

22. Nakajima M, Cansay E, Senawomg P, Ozer F, Ogata M, and Tagami J. Microtensile bond strength of filled vs unfilled adhesive to dentin using self-etch and totaetch technique. J Dent 2006;34:283-291.

23. Chersoni S, Suppa P, Grandini S, Goracci C, Monticelli F, Yiu C, Huang C, Prati C, Breschi L, Ferrari M, Pashley DH, and Tay FR. In vivo and in vitro permeability of one-step self-etch adhesives, J Dent Res 2004;83:459-464. 